Bull. Austral. Math. Soc.

$34 \mathrm{C} 25,34 \mathrm{C} 15,58 \mathrm{~F} 22,70 \mathrm{~F} 10$

VoL. 58 (1998) [1-13]

\title{
MULTIPLE CLOSED ORBITS FOR $N$-BODY-TYPE PROBLEMS
}

\author{
SHIQING ZHANG
}

\begin{abstract}
Using the equivariant Ljusternik-Schnirelmann theory and the estimate of the upper bound of the critical value and lower bound for the collision solutions, we obtain some new results in the large concerning multiple geometrically distinct periodic solutions of fixed energy for a class of planar $N$-body type problems.
\end{abstract}

\section{INTRODUCTION}

In recent years, some researchers have applied variational methods to the study of the existence and multiplicity of periodic solutions for certain classes of $N$-body-type problems (see for example [3]). But there have been few results in the large concerning the existence of multiple geometrically distinct periodic solutions of fixed energy. The $N$-body problem in $\mathbf{R}^{\mathbf{k}}$ has $S^{1} \times O(k)$ symmetry (where $O(k)$ is the rotational symmetry group of order $k$ ). In order to obtain multiple geometrically distinct periodic solutions, we must consider the effects of the group $S^{1} \times O(k)$. Using the equivariant Ljusternik-Schnirelmann theory and precise estimates on the collision solutions and critical values for the variational functional, we obtain some new results in the large concerning with multiple geometrically distinct periodic solutions of fixed energy for some planar $N$-body problems including planar $N$-body problems with Keplerian potentials. Under certain conditions, we shall show in Theorem 1.3 that there exist at least $2(N-1) 2^{N-2}$ geometrically distinct noncollision periodic trajectories for the system $(\mathrm{Ph})$ as defined below. It should be noted that the authors are unaware of any published results on the existence of multiple geometrically distinct periodic solutions for $N$-body problem with Keplerian type weak force potentials.

We set $\Omega=\mathbf{R}^{2} /\{0\}$ and consider a potential $V$ of the form:

$$
V(u)=V\left(u_{1}, \ldots, u_{N}\right)=\frac{1}{2} \sum_{1 \leqslant i \neq j \leqslant N} V_{i j}\left(u_{i}-u_{j}\right)
$$

Received 17th March, 1997

The author would like to thank the referee for useful suggestions and thanks Ms. Cindy White for typing this paper.

Copyright Clearance Centre, Inc. Serial-fee code: 0004-9729/98 \$A2.00+0.00. 
where $u_{i} \in \mathbf{R}^{2}, u=\left(\mathbf{u}_{1}, \ldots, \mathbf{u}_{\mathbf{N}}\right) \in\left(\mathbf{R}^{2}\right)^{\mathbf{N}}$ and $V_{i j} \in C^{1}(\Omega, \mathbf{R})$. Given $T>0$, we seek periodic solutions of $(\mathrm{Ph})$ :

$$
\begin{gathered}
m^{i} \frac{d^{2} x_{i}}{d t^{2}}+\frac{\partial}{\partial x_{i}} V\left(x_{1}, \ldots, x_{N}\right)=0, \quad 1 \leqslant i \leqslant N \\
\frac{1}{2} \sum_{i=1}^{N} m_{i}\left|\dot{x}_{i}(t)\right|^{2}+V\left(x_{1}(t), \ldots, x_{N}(t)\right)=h .
\end{gathered}
$$

Definition 1.1: By a solution of $(\mathrm{Ph})$, we mean $x(t)=\left(x_{1}(t), \ldots, X_{N}(t)\right)$ such that $x$ is periodic with period $T>0$ for all $i, j=1, \ldots, N$ satisfying:

(i) $x_{i} \in H^{1,2}\left(0, T ; \mathbf{R}^{2}\right)$;

(ii) the set $S=\left\{t \in[0, T] \mid x_{i}(t)=x_{j}(t)\right.$ for some $\left.i \neq j\right\}$ has measure zero;

(iii) $x_{i}$ is $C^{2}$ on $[0, T) / S$ and satisfies $(\mathrm{Ph})$.

A solution $x$ such that $S \neq \emptyset$ (respectively $=\emptyset$ ) is called a collision (respectively noncollision) solution.

DEFinition 1.2: Let $x$ and $y$ be two periodic solutions of (Ph). If there are diffeomorphisms $\varphi: S^{1} \rightarrow S^{1}$ and $R: O(2) \rightarrow O(2)$ such that $y=R \cdot x \cdot \varphi$, then we call $x$ and $y$ distinct in geometry.

REMARK. $x$ and $y$ are the same in geometry is equivalent to that $x$ and $y$ belong to the same orbit and have the same period so that there are $\theta \in S^{1}$ and $R \in O(2)$ such that $y(t)=R x(t+\theta)$.

Theorem 1.3. Assume $V$ possesses the form of (1.1) and $V_{i j}(i, j=1,2, \ldots, N)$ satisfy, for $i \neq j$, the following conditions:

(V1) $\quad V_{i j}(\zeta)=V_{j i}(\zeta)$ for all $\zeta \neq 0$

(V2) $\exists \alpha \in[1,2)$ such that $V_{i j}^{\prime}(\zeta) \zeta \geqslant-\alpha V_{i j}(\zeta)>0, \forall \zeta \neq 0$;

(V3) $\exists \beta \in[0,2)$ and $r>0$ such that $V_{i j}^{\prime}(\zeta) \zeta \leqslant-\beta V_{l i j}(\zeta), \forall 0<|\zeta| \leqslant r$;

(V4) $\quad V_{i j}(\zeta) \rightarrow 0$ as $|\zeta| \rightarrow+\infty$;

(V5) $3 V_{i j}^{\prime}(\zeta) \zeta+V_{i j}^{\prime \prime}(\zeta) \zeta \cdot \zeta>0$, for all $\zeta \neq 0$;

(V6) $\exists a, b>0$ such that

$$
\frac{a}{2} \sum_{1 \leqslant i \neq j \leqslant N} \frac{m_{i} m_{j}}{\left|x_{i}-x_{j}\right|^{\alpha}} \leqslant-V(x) \leqslant \frac{b}{2} \sum_{1 \leqslant i \neq j \leqslant N} \frac{m_{i} m_{j}}{\left|x_{i}-x_{j}\right|^{\alpha}}
$$


(V7) $C<\min \left\{C_{1}, 9 C_{3}\right\}$ where

$$
\begin{aligned}
C & =D^{2}\left(\sum_{i-1}^{N} \pi^{2} m_{i} \cos ^{2} \frac{\pi i}{2 N}\right) \cdot\left(\frac{2 \beta}{2-\beta}\right) \\
D & =\left[b\left(\frac{1}{2}-\frac{\alpha}{4}\right) \sum_{i \leqslant i \neq j \leqslant N} \frac{m_{i} m_{j}}{\left|\cos \frac{\pi \cdot i}{2 N}-\cos \frac{\pi \cdot j}{2 N}\right|^{\alpha}}\right]^{1 / \alpha} \\
C_{1} & =\min _{2 \leqslant s \leqslant N}\left\{\pi \gamma^{2}\left(\frac{a}{2}\right)^{2 / \alpha} K_{s}\right\} \\
C_{2} & =2 B M^{-\alpha /(2+\alpha)} \sum_{1 \leqslant i \neq j \leqslant N} m_{i} m_{j}, C_{3}=\frac{1}{4}\left(\frac{2 \beta}{2-\beta}\right)^{(\alpha-2) / \alpha} \cdot C_{2}^{(2+\alpha) / \alpha} \\
\gamma & =\frac{\Gamma\left(\frac{1}{\alpha}-\frac{1}{2}\right)}{\Gamma\left(\frac{1}{\alpha}\right)} \\
B & =2^{-2 \alpha /(\alpha+2)} \cdot A \\
A & =4 \pi^{2}(1+2 / \alpha) \cdot\left[\frac{\alpha^{2} a}{(2 \pi)^{2} 2^{(8+\alpha) / 2}}\right]^{2 /(2+\alpha)} \\
M & =\sum_{i=1}^{N} m_{i} \\
\Gamma(x) & =\int_{0}^{\infty} t^{x-1} \varepsilon^{-t} d t, K_{s}-\min _{\tau \in S_{n}} \frac{\left(\sum_{i \leqslant \neq j \leqslant s} m_{\tau(i)} m_{\tau(j)}\right)^{(2+\alpha) / \alpha}}{\sum_{i=1}^{s} m_{\tau(i)}}
\end{aligned}
$$

(where $S_{N}$ denotes the group of all the permutations of $\{1, \ldots, N\}$ ).

Then for any $h<0$, system $(\mathrm{Ph})$ has at least $2(N-1) \cdot 2^{N-2}$ geometrically distinct noncollision periodic solutions.

Corollary 1.4. Assume $N=2, V_{i j}(\zeta)=-1 /|\zeta|$, that is, $m_{1}=m_{2}=1, \alpha=$ $\beta=1, a=b=1$. Then for any $h<0$, system $(\mathrm{Ph})$ has at least two geometrically distinct noncollision periodic solutions.

Proof: By the assumptions we have $M=2, A+3 \cdot 2^{-3} \cdot(2 \pi)^{2 / 3}, B=3 \cdot 2^{-3}$. $\pi^{2 / 3}, C_{2}=3 \cdot 2^{-4 / 3} \cdot \pi^{2 / 3}, C_{3}=27 / 128 ; \gamma=\Gamma(1 / 2) / \Gamma(1)=\pi^{1 / 2}, K_{2}=4, C_{1}=$ $\pi^{2}, D=\sqrt{2} / 2, C=\pi^{2} / 2$. Hence $C<C_{1}$ and $C<9 C_{3}$.

\section{The PRoOF of the MAIN ReSUlts}

Let us introduce the following notations:

$$
H=W^{1,2}\left(S^{1}, \mathbf{R}^{2}\right)
$$




$$
\begin{aligned}
& H_{\#}=\left\{u \in H \mid u\left(t+\frac{1}{2}\right)=-u(t)\right\} \\
& E=\left\{u=\left(u_{1}, \ldots, u_{n}\right) \mid u_{i} \in H_{\#}, i=1, \ldots, N\right\} \\
& \Lambda_{0}=\left\{u \in E \mid u_{i}(t) \neq u_{j}(t), \forall t \in \mathbf{R}, i \neq j\right\} \\
& \|u\|_{E}^{2}=\sum_{i=1}^{N} m_{i}\left\|u_{i}\right\|^{2}=\sum_{i=1}^{N} m_{i} \int_{0}^{1}\left|\dot{u}_{i}\right|^{2} d t, \forall u=\left(u_{1}, \ldots, u_{N}\right) \in E .
\end{aligned}
$$

Define the subset $M$ of $\Lambda_{0}$ and the following functional $f$ on $M$ :

$$
\begin{aligned}
M & =\left\{u \in \Lambda_{0} \mid \int_{0}^{1}\left[V(u)+\frac{1}{2} V^{\prime}(u) u\right] d t=h\right\} \\
f(u) & =\frac{1}{4}\|u\|_{E}^{2} \cdot \int_{0}^{1} V^{\prime}(u) u d t .
\end{aligned}
$$

The following lemma whose proofs can be found in $[2,8]$ gives sense to these definitions.

LEMMA 2.1. Let $h<0$, and let $V_{i j}$ satisfy (V1)-(V5). Then

(i) $M \neq \emptyset, M$ is a $C^{\infty}$ manifold in $E$ and a strong deformation retract of $\Lambda_{0}$.

(ii) Let $u$ be a non-constant critical point of $\left.f\right|_{M}$; also let

$$
\frac{1}{T^{2}}=\frac{\int_{0}^{1} V^{\prime}(u) u d t}{\|u\|_{E}^{2}}
$$

Then $x(t)=u(t / T)$ is a $T$-periodic noncollision solution of (Ph); conversely if $x(t)$ is a $C^{2}$ solution of $(P h)$ of period $T$, then $u(t)=x(T t)$ is critical point of $\left.f\right|_{M}$.

LEMMA 2. 2 .

(i) $\int_{0}^{T} \frac{1}{|q(t)|^{\alpha}} d t \geqslant T^{(2+\alpha) / 2}\left(\int_{0}^{T}|q(t)|^{2} d t\right)^{-\alpha / 2}$.

(ii) $\int_{0}^{T}\left(\sum_{i=1}^{N} m_{i}\left|u_{i}\right|^{2}\right)^{-\alpha / 2} d t \geqslant T^{1+(\alpha / 2)}\left[\int_{0}^{T}\left(\sum_{i=1}^{N} m_{i}\left|u_{1}\right|^{2}\right) d t\right]^{-\alpha / 2}$.

(iii) (Wirtinger Inequality) $\|\dot{u}\|_{2} \geqslant \frac{2 \pi\|u\|_{2}}{T}, \quad \forall u \in H_{\#}$.

ProOF: (i) By Hölder's inequality, we have

$$
\int_{0}^{T}|q| d t \leqslant T^{1 / 2}\left(\int_{0}^{T}|q|^{2} d t\right)^{1 / 2},\left(\int_{0}^{T}|q|^{2} d t\right)^{\alpha / 2} \geqslant T^{-\alpha / 2}\left(\int_{0}^{t}|q| d t\right)^{\alpha} .
$$


By Chebychev's inequality, we have

$$
\int_{0}^{T} \frac{1}{|q|} d t \cdot \int_{0}^{T}|q|^{2} d t \geqslant T^{2}
$$

So

$$
\left(\int_{0}^{T}|q|^{-1} d t\right)^{\alpha} \geqslant T^{3 \alpha / 2}\left(\int_{0}^{T}|q|^{2} d t\right)^{-\alpha / 2}
$$

Using the above results and Jensen inequality, we have

$$
\int_{0}^{T}|q|^{-\alpha} d t \geqslant T\left(\frac{1}{T} \int_{0}^{T}|q|^{-1} d t\right)^{\alpha} \geqslant T^{1+(\alpha / 2)}\left(\int_{0}^{T}|q|^{2} d t\right)^{-\alpha / 2}
$$

The proof of inequality (ii) is similar to that of (i).

LEMMA 2.3. Let $K=\inf \left\{\lim _{n \rightarrow \infty} \inf f\left(u_{m}\right) \mid\left\{u_{n}\right\} \subset M, u_{n} \stackrel{w}{\longrightarrow} u \in \partial \Lambda_{0}\right\}$. Then, $\forall \varepsilon>0$, the (P.S.) condition holds in $f^{K-\varepsilon}=\{u \in M \mid f(u) \leqslant K-\varepsilon\}$.

Proof: Let $\left\{u_{n}\right\}$ be a (P.S.) sequence at level $c<K$. For $F\left(u_{n}\right) \rightarrow c$, we know that $\int_{0}^{T}\left|\dot{u}_{n}\right|^{2} d t$ is bounded. Thus $\left\{u_{n}\right\}$ is bounded in $E$. Taking a subsequence if necessary, we can say $u_{n} \stackrel{w}{\rightarrow} u$. For $c<K$ we have the $u \in \Lambda_{0}$. Similar to the proof of [1] or [4], $f\left(u_{n}\right) \rightarrow 0$ and $u_{n} \stackrel{w}{\longrightarrow} u \in \Lambda_{0}$ imply $u_{n} \stackrel{s}{\longrightarrow} u \in M$.

Moreover $\forall k<K, f^{k}$ is complete. In the following, we derive an estimate from above on minimax levels on a special set with large $S^{\mathbf{1}} \times O(2)$ Ljusternik-Schnirelmann category.

Similar to the proof of [2], if (V1)-(V5) hold, there exists a continuous function $d: \Lambda_{0} \rightarrow R$ such that $d(u) u \in M, \forall u \in \Lambda_{0}$. In fact, we set for $u \in \Lambda_{0}$ and $d>0$

$$
\emptyset(d)=\int_{0}^{1}\left[V(d u)+\frac{1}{2} V^{\prime}(d u) d u\right] d t
$$

From (V1)-(V5), it follows that

$$
\begin{aligned}
\emptyset^{\prime}(d)>0 & \text { for all } d \in(0, \infty) \\
\emptyset(d) \rightarrow-\infty & \text { as } d \rightarrow 0 \\
\emptyset(d) \rightarrow 0 & \text { as } d \rightarrow \infty
\end{aligned}
$$

Thus there is a unique $d=d(u) \in C\left(\Lambda_{0}, \mathbf{R}\right)$ such that $\emptyset(d)=h$ for all $u \in \Lambda_{0}$ and $h<0$. Thus a possible homotopy retracting $H$ on $M$ has the for $H(\lambda, u)=$ 
$(1-\lambda) u+\lambda d(u) u$. For $i=1, \ldots, N$, let

$$
\begin{array}{ll}
Z_{i}=\left\{v(t)=\left(\cos \frac{\pi i}{2 N}\right)(\zeta \cos 2 \pi t+\eta \sin 2 \pi t) \mid \zeta, \eta \in \mathbf{R}^{2},\right. \\
\text { (12) } \quad|\zeta|=|\eta|=1,\langle\zeta, \eta\rangle=0\} . \\
\text { (13) } Z=Z_{1} \times \cdots \times Z_{N}, \widetilde{Z}=H(1, Z) \subset M .
\end{array}
$$

In the following we will estimate the upper bound of $f$ on $\tilde{Z}$, for this, we note that for any $u=\left(u_{1}, \ldots, u_{N}\right) \in Z$, there are $\zeta_{i}, \eta_{i} \in \mathbf{R}^{2}$ such that

$$
|\zeta|=\left|\eta_{i}\right|=1, \zeta_{i} \eta_{i}=0, u_{i}=\left(\cos \frac{\pi i}{2 N}\right)\left(\zeta_{i} \cos 2 \pi t+\eta_{i} \sin 2 \pi t\right) \text {. }
$$

LEMMA 2.4. If $V$ satisfies (V6), then

$$
-V(u) \leqslant \frac{b}{2} \sum_{i \neq j} \frac{m_{i} m_{j}}{\left|\cos \frac{\pi i}{2 N}-\cos \frac{\pi i}{2 N}\right|^{\alpha}} .
$$

Proof: By $\left|u_{i}(t)\right|^{2}=(\cos (\pi i) /(2 N))^{2}$ we have

$$
\begin{aligned}
\left|u_{i}(t)-u_{j}(t)\right|^{2} & \geqslant\left|u_{i}(t)\right|^{2}+\left|u_{j}(t)\right|^{2}-2\left|u_{i}(t)\right| \cdot\left|u_{j}(t)\right| \\
& \geqslant \cos ^{2} \frac{\pi i}{2 N}+\cos ^{2} \frac{\pi j}{2 N}-2 \cos \frac{\pi i}{2 N} \cdot \cos \frac{\pi j}{2 N}=\left(\cos \frac{\pi i}{2 N}-\cos \frac{\pi j}{2 N}\right)^{2} .
\end{aligned}
$$

Hence the lemma is proved by (V6).

In the following, we will use Lemma 2.4 to estimate the bound from above on the functional $f$ constrained on $\widetilde{Z}$.

The following lemma is easily proved

LEMmA 2.5. Let $V_{i j}$ satisfy $(V)-(V 6)$, then for any real number $d$ and any fixed $u \in E$, the functional $f(d u)$ in an increasing functional on $d$.

By Lemma 2.4 and Lemma 2.5, we have

LEMмA 2.6. If $V_{i j}$ satisfies (V1)-(V6), then we have

$$
\begin{aligned}
d(u) & \leqslant\left[b\left(\frac{1}{2}-\frac{1}{4 \alpha}\right) \sum_{i \neq j} \frac{m_{i} m_{j}}{\left|\cos \frac{\pi}{2 N}-\cos \frac{\pi j}{2 N}\right|^{\alpha}}\right]^{1 / \alpha}(-h)^{-1 / \alpha} \\
& \equiv D(-h)^{-1 / \alpha}, \forall u \in Z \\
k & =\max _{\bar{u} \in \bar{Z}} f(u) \leqslant C \cdot(-h)^{1-2 / \alpha}
\end{aligned}
$$

where

$$
C=D^{2}\left(\sum_{i=1}^{N} \pi^{2} m_{i} \cos ^{2} \frac{\pi i}{2 N}\right) \cdot \frac{2 \beta}{2-\beta}
$$


ProOF: By (V1)-(V6) and Lemma 2.4, we have

$$
\begin{aligned}
h & =\int_{0}^{1}\left[V(d \cdot u)+\frac{1}{2} V^{\prime}(d \cdot u) d u\right] d t \geqslant\left(1-\frac{\alpha}{2}\right) \int_{0}^{1} V(d \cdot u) d t \\
& \geqslant \frac{1}{2}\left(1-\frac{\alpha}{2}\right) \int_{0}^{1} \frac{1}{d^{\alpha}(u)} \cdot \sum_{i \neq j} \frac{-b m_{i} m_{j}}{\left|u_{i}(t)-u_{j}(t)\right|^{\alpha}} d t \\
& \geqslant-\frac{b}{2}\left(1-\frac{\alpha}{2}\right)(d(u))^{-\alpha} \sum_{i \neq j} \frac{m_{i} m_{j}}{\left|\cos \frac{\pi i}{2 N}-\cos \frac{\pi i}{2 N}\right|^{\alpha}} .
\end{aligned}
$$

So the estimate (17) on $d(u)$ holds.

We take $\bar{u} \in \widetilde{Z}$, as $\bar{u}=d(u) u$ with $u \in Z$ and $d(u) \leqslant D(-h)^{-1 / \alpha}$. Hence by $\left|u_{i}\right|=\cos (\pi i) /(2 N)$, we have

$$
\begin{aligned}
\|\bar{u}\|_{E}^{2} & =\sum_{i=1}^{N} m_{i} \int_{0}^{1}\left|\bar{u}_{i}^{\prime}\right|^{2} d t=\sum_{i=1}^{N} m_{i} D^{2}\left(4 \pi^{2} \int_{0}^{1}\left|u_{i}\right|^{2} d t\right) \cdot(-h)^{-2 / \alpha} \\
& =4 \pi^{2} D^{2}\left(\sum_{i=1}^{N}\left(\cos \frac{\pi i}{2 N}\right)^{2} m_{i}\right) \cdot(-h)^{2 / \alpha}
\end{aligned}
$$

By (7) and (V3), we have

$$
\int_{0}^{I} V^{\prime}(\widetilde{u}) \tilde{u} d t \leqslant \frac{-2 \beta h}{2-\beta} .
$$

So the upper bound estimate (18) is proved by (8).

Similar to [4] and [12], we have

LEMMA 2.7. Let $u \in M$ and let $1 / T^{2}=\left(\int_{0}^{1} V^{\prime}(u) u d t\right) /\|u\|_{E}^{2}$, also let $q(t)=$ $u(t / T)$. Then the following equalities hold:

$$
\begin{aligned}
& {[4 f(u)]^{1 / 2}=\int_{0}^{T}\left[\frac{1}{2} \sum_{i=1}^{N} m_{i}\left|\dot{q}_{i}\right|^{2}+\frac{1}{2} V^{\prime}(q) q\right] d t} \\
& {[4 f(u)]^{1 / 2}=T \int_{0}^{1} V^{\prime}(u) u d t .}
\end{aligned}
$$

Proof: By (8) and (9) we have

$$
[4 f(u)]^{1 / 2}=\|u\|_{E} \cdot\left(\int_{0}^{1} V^{\prime}(u) u d t\right)^{1 / 2}=T \int_{0}^{1} V^{\prime}(u) u d t
$$


Hence (21) is proved. By (8) and (9) we have

$$
\begin{aligned}
f(u) & =\frac{1}{4}\|u\|_{E}^{2} \cdot \int_{0}^{1} V^{\prime}(u) u d t=\frac{1}{4 T}\|u\|_{E}^{2} \cdot \int_{0}^{T} V^{\prime}\left(u\left(\frac{\tau}{T}\right)\right) u\left(\frac{\tau}{T}\right) d t \\
& =\frac{T}{4} \int_{0}^{1} V^{\prime}(u) u d t \cdot \int_{0}^{T} V^{\prime}(q) q d \tau=\frac{1}{4}\left(\int_{0}^{T} V^{\prime}(q) q d t\right)^{2}[4 f(u)]^{1 / 2} \\
& =\int_{0}^{T} V^{\prime}(q) q d t .
\end{aligned}
$$

On the other hand, by (8) and (9) we have

$$
[4 f(u)]^{1 / 2}=\|u\|_{E} \cdot\left(\int_{0}^{1} V^{\prime}(u) u d t\right)^{1 / 2}=\frac{1}{T}\|u\|_{E}^{2}=\frac{1}{T} \int_{0}^{1} \sum_{i=1}^{N} m_{i}\left|\dot{u}_{i}(t)\right|^{2} d t .
$$

So

$$
[4 f(u)]^{1 / 2} \int_{0}^{T} \sum m_{i}\left|\dot{q}_{i}\right|^{2} d t
$$

Adding up (22) and (23), we have

$$
[f(u)]^{1 / 2}=\int_{0}^{T} \frac{1}{2}\left[\sum_{i=1}^{N} m_{i}\left|\dot{q}_{i}\right|^{2}+V^{\prime}(q) \cdot q\right] d t .
$$

We denote $E_{1}=\left\{u \in E \mid \int_{0}^{1}-V(u) d t<\infty\right\}$. Then, by (V3), for all $u \in E_{1}$, we have also $\int_{0}^{1} V^{\prime}(u) u d t<\infty$. We set

$$
M_{1}=\left\{u \in E_{1}=\int_{0}^{1}\left(V(u)+\frac{1}{2} V^{\prime}(u) u\right) d t=h\right\} .
$$

We also denote, for $u \in E_{1}$,

$$
f(u)=\frac{1}{2}\|u\|^{2} \cdot \int_{0}^{1}[h-V(u)] d t .
$$

Now it is easy to see that from (V1)-(V6) it follows that for all $u \in E_{1}$ we have $\{\lambda u, \lambda>0\} \subset E_{1}$ and there exists a unique $\lambda(u)>0$ such that $\lambda(u) u \in M_{1}$ and

$$
I(u) \equiv \max _{\lambda>0} f(\lambda u)=f(\lambda(u) u) .
$$

We set, for $s=2, \ldots, N$,

$$
\partial \tilde{\Lambda}_{s}=\left\{U \in E_{1}: \exists \tau \in S_{N}, \exists \bar{t} \in S^{1}: u_{\tau(1)}(\bar{t})=\cdots=u_{\tau(s)}(\bar{t})\right\}
$$


where $S_{N}$ denotes the group of all permutations of $\{1, \ldots, N\}$.

LEMMA 2.8. [11]. Let

$$
\gamma=\frac{\Gamma\left(\frac{1}{\alpha}-\frac{1}{2}\right)}{\Gamma\left(\frac{1}{\alpha}\right)}
$$

and

$$
K_{s}=\min _{\tau \in S_{N}} \frac{\left(\sum_{1 \leqslant i \neq j \leqslant s} m_{\tau(i)} m_{\tau(j)}\right)^{(2+\alpha) / \alpha}}{\sum_{i=1}^{s} m_{\tau(i)}} .
$$

Then

$$
\inf _{u \in M_{1} \cap \partial \widetilde{\Lambda}_{s}} f(u)=\inf _{u \in \partial \widetilde{\Lambda}_{s}} I(u) \geqslant \pi \gamma^{2}\left(\frac{\alpha}{2}\right)^{2 / \alpha} \cdot K_{s} \cdot|h|^{(\alpha-2) / \alpha} .
$$

LEMma 2.9. If $V$ satisfies (V1)-(V6), then $K>C_{1}|h|^{(\alpha-2) / a}$, where $K$ is defined in Lemma 2.3, $C_{1}$ is defined in Theorem 1.3.

Proof: By the definition of $K$, we take a sequence $\left\{u_{n}\right\} \in M$ such that $u_{n} \stackrel{w}{\longrightarrow} u \in$ $\partial \Lambda_{0}$ and $f\left(u_{n}\right) \rightarrow K$. Then $u$ is not a constant. Suppose for a contradiction that $u(t) \equiv$ constant. By the symmetry $u(t+1 / 2)=-u(t)$, we have $u(t) \equiv 0$, then $u_{n} \stackrel{w}{\longrightarrow} 0$. By the Sobolev embedding theorem, we have $\left\|u_{n}\right\| \rightarrow 0$ and $\int_{0}^{1} V\left(u_{n}\right) d t \rightarrow-\infty$. But by (V2) and (V3), we have

$$
\begin{aligned}
\left(1-\frac{\alpha}{2}\right) \int_{0}^{1} V\left(u_{n}\right) d t \leqslant h=\int_{0}^{1}\left[V\left(u_{n}\right)+\frac{1}{2} V^{\prime}\left(u_{n}\right) u_{n}\right] d t & \\
& \leqslant\left(1-\frac{\beta}{2}\right) \int_{0}^{1} V\left(u_{n}\right) d t .
\end{aligned}
$$

This is a contradiction. Hence, $u$ is not a constant. Let $\partial \Lambda_{s}=\{u \in E: \exists \tau \in$ $\left.S_{N}, \exists \bar{t} \in S^{1}=u_{\tau(1)}(\bar{t})=\cdots=u_{\tau(s)}(\bar{t})\right\}$. By the same procedure as in the proof of [11, Lemma 2.8], we can obtain

$$
\inf _{u \in M \cap \partial \Lambda} f(u) \geqslant \pi \gamma^{2}\left(\frac{\alpha}{2}\right)^{2 / \alpha} \cdot K_{s} \cdot|h|^{(\alpha-2) / \alpha} .
$$

By $\partial \Lambda_{0}=\bigcup_{s=2}^{N} \partial \Lambda_{s}$, we have

$$
K \geqslant \inf _{u \in M \cap \partial \Lambda_{0}} f(u) \geqslant \min _{2 \leqslant s \leqslant N}\left\{\pi \gamma^{2}\left(\frac{\alpha}{2}\right)^{2 / \alpha} K_{s}\right\} \cdot|h|^{(\alpha-2) / \alpha}=C_{1}|h|^{(\alpha-2) / \alpha}
$$


LEMMA 2.10. If $u$ is a critical point of $\left.f\right|_{M}$, and (V1)-(V6) hold, then we have

$$
f(u) \geqslant C_{3}(-h)^{(\alpha-2) / \alpha} \text {. }
$$

Proof: As in Lemma 2.7, let $T^{2}=\|u\|_{E}^{u} /\left(\int_{0}^{1} V^{\prime}(u) u d t\right)$ and $q(t)=u(t / T)$. Then

$$
\left[4 f(u)^{1 / 2}\right]=\int_{0}^{1}\left[\frac{1}{2} \sum_{i=1}^{N} m_{i}\left|\dot{q}_{i}\right|^{2}+\frac{1}{2} V^{\prime}(q) q\right] d t
$$

Using the symmetry property $q(t+(T / 2))=-q(t)$ and (V6), we deduce that

$$
[4 f(u)]^{1 / 2} \geqslant \sum_{i=1}^{N} \frac{m_{i}}{2} \int_{0}^{T}\left|\dot{q}_{i}\right|^{2} d t+\frac{\alpha a}{4} \int_{0}^{T} \frac{d t}{\left|q_{i}-q_{j}\right|^{\alpha}}
$$

Using the methods of [6] and [8], we can give more explicit estimates about $[4 f(u)]^{1 / 2}$. Let

$$
\begin{aligned}
M=\sum_{i=1}^{N} m_{i}, A & =4 \pi^{2}\left(1+\frac{2}{\alpha}\right)\left[\frac{\alpha^{2} a}{(2 \pi)^{2} 2^{(8+\alpha) / 2}}\right]^{2 /(2+\alpha)} \\
B & =2^{2 \alpha /(\alpha+2)} \cdot A
\end{aligned}
$$

Then

$$
[4 f(u)]^{1 / 2} \geqslant 2 B M^{-\alpha /(2+\alpha)} \sum_{1 \leqslant i \neq j \leqslant N} m_{i} m_{j}=C_{2} T^{(2-\alpha) /(2+\alpha)}
$$

On the other hand, for every $u \in M$, we have $\int_{0}^{1}\left[V(u)+V^{\prime}(u) u / 2\right] d t=h$. From this and (V3) we deduce

$$
\int_{0}^{1} V^{\prime}(u) u d t \leqslant \frac{-2 \beta h}{2-\beta}, \quad \forall u \in M .
$$

By (21) and (34), we get

$$
[4 f(u)]^{1 / 2} \leqslant T\left(\frac{-2 \beta h}{2-\beta}\right)
$$

Plugging (35) into (33), we get

$$
T^{2 \alpha /(2+\beta)}\left(\frac{-2 \beta h}{2-\beta}\right) \geqslant C_{2}
$$


From (35) and (36), it follows that

$$
f(u) \geqslant \frac{1}{4}\left(\frac{-2 \beta h}{2-\beta}\right)^{(\alpha-2) / \alpha} \cdot C_{2}^{(2+\alpha) / \alpha} \equiv C_{3} \cdot(-h)^{(\alpha-2) / \alpha} .
$$

We note that $Z$ is $S^{1} \times O(2)$ invariant. In fact, for any $x(t)=\left(x_{1}(t), \ldots, x_{N}(t)\right)$ and $\tau \in S^{1}, R \in O(2), H(\tau, R) \cdot x(t) \equiv\left(R x_{1}(t+\tau), \ldots, R x_{N}(t+\tau)\right) \in Z$. We also note that $\widetilde{Z}$ is $S^{1} \times O(2)$ invariant and the functional $f$ is $S^{1} \times O(2)$ invariant. Hence in order to obtain multiple distinct $S^{1} \times O(2)$ invariant orbits of the system $(\mathrm{Ph})$, we need to estimate the $S^{1} \times O(2)$ equivariant category of the special sets $Z$ and $\widetilde{Z}$. We note that each $Z_{i}(i=1, \ldots, N)$ is diffeomorphic to $T_{1} S^{1}$ which is the unit tangent bundle of $S^{1}$. Hence $\operatorname{cat}\left(Z_{i}\right) \geqslant 3$. Now $Z=Z_{1} \times \cdots \times Z_{N}$ is diffeomorphic to $T_{1} S^{1} \otimes \cdots \otimes T_{1} S^{1}$ ( $N$ factors), and $Z$ is diffeomorphic to the set $Z_{\left(n_{1}, \ldots, n_{N}\right)}$ of Coti Zelati [5], so by [5], we have

LEMMA 2.11. $i(Z) \equiv \operatorname{cat}\left(Z / S^{1} \times O(2)\right) \geqslant 2(N-1) 2^{N-2}$.

REMARK. Although the set $Z$ is diffeomorphic to the critical manifold $Z_{\left(n_{1}, \ldots, n_{N}\right)}$ of Coti Zelati [5], the upper bounds of $f$ on $Z$ and $\widetilde{Z}$ are more easily estimated than on $Z_{\left(n_{1}, \ldots, n_{N}\right)}$.

Lemma 2.12. [10] Let $X$ and $Y$ be two arcwise connected topological spaces. If the continuous map $\varphi: X \rightarrow Y$ has a left inverse $\psi: Y \rightarrow X$, then $\forall A \subseteq$ $X, \operatorname{Cat}_{Y}(\varphi(A)) \geqslant \operatorname{cat}_{X}(A)$.

The following lemma is an equivariant version of Ljusternik-Schnirelmann theory.

Lemma 2.13. Let $X$ be a Banach space, $\Lambda$ an open subset of $X$, and $f \in$ $C^{1}(\Lambda, \mathbf{R})$. Let $G$ be a compact Lie group, $T(G)$ a linear continuous representation with equivariant distance and $M$ a $C^{2-0}$ submanifold of $\Lambda$. Assume $M$ and $f$ both are invariant under $T(G), f$ satisfies the Palais-Smale condition on some closed subset of $N$ of $M$.

Let $i$ be a $T(G)$-invariant index. Let $c_{m}=\inf _{i(A) \geqslant(m)} \operatorname{Sup}_{x \in Z} f(x), m=1,2, \ldots$, where $A \subset N, A \in \sum=\{B \subset N \mid B$ is $T(G)$-invariant and closed in $N\}$. Then

(1) when $-\infty<c_{m}<+\infty, c_{m}$ is critical value of $f$,

(2) if $-\infty<c=c_{m+1}=\cdots=c_{m+k}<+\infty$, then $i\left(K_{c}\right) \geqslant k$, where $K_{c}=\left\{x \in N \mid f^{\prime}(x)=0, f(x)=c\right\}$,

(3) $c_{m} \leqslant c_{m+1}$.

LEMMA 2.14. If $u$ is a critical point of $\left.f\right|_{M}$, which has minimal period $1 / l, l \neq$ 1 , then $l \geqslant 3$ and $v(t)=u(t / l)$ is also a critical point of $\left.f\right|_{M}$ and $f(v)=\left(1 / l^{2}\right) f(u) \leqslant$ $f(u) / 9$. 
Proof: The result follows from the symmetry $u(t+1 / 2)=-u(t)$ and the following two equalities:

$$
\int_{0}^{T}|\dot{v}(t)|^{2} d t=\frac{1}{l^{2}} \int_{0}^{T}|\dot{u}(t)|^{2} d t ; \int_{0}^{T} V(v) \cdot v d t=\int_{0}^{T} V(u) u d t
$$

We are now ready to prove Theorem 13.

Proof: Inequality $C_{1}>C$ implies that $K>k$. Thus by Lemma 2.3, the PalaisSmale condition holds in $f^{k}$. We note that $\widetilde{Z} \subset f^{k} \subset M$ by the definition of $k$. Thus by Lemma 2.11 and Lemma 2.12 , we have $i(\tilde{Z})(\tilde{Z}) \geqslant i_{Z}(Z)=2(N-1) 2^{N-2}$. Since $i_{(\tilde{Z})}(\tilde{Z}) \geqslant 2(N-1) 2^{N-2}$ and (P.S.) condition holds in $f^{k}$, we are in a position to apply the $S^{1} \times O(2)$-equivariant Ljusternik-Schnirelmann theory in $N=f^{k}$, which yields the existence of $2(N-1) 2^{N-2}$ critical points $\left\{u_{i} \mid i=1, \ldots, 2(N-1) 2^{N-2}\right\} \subset f^{k}$ of $\left.F\right|_{M}$, none of which can be brought into the other by the standard $S^{1} \times O(2)$ action of $M$. For any $u_{1} \in f^{k}$ we have $f\left(u_{i}\right) \leqslant C(-h)^{1-2 / \alpha}$. If $u_{i}$ were not of minimal period 1 , from Lemma 2.14, it would follow that there is a critical point $v_{i}$ of $\left.f\right|_{M}$ such that $f\left(v_{i}\right) \leqslant(C / 9)(-h)^{1-2 / \alpha}$. Lemma 2.10 and estimate $C_{3}>C / 9$ would then imply a contradiction, so Theorem 1.3 is proved.

\section{REFERENCES}

[1] A. Ambrosetti and V. Coti Zelati, 'Closed orbits of fixed energy for a class of $N$-body problems', Ann. Inst. H. Poincaré Anal. Non Linéaire 9 (1992), 187-200; (Addendum to closed orbits of fixed energy for a class of $N$-problems, Ann. Inst. H. Poincaré Analyse Nonlineaire 9 (1992), 337-338).

[2] A. Ambrosetti and V. Coti Zelati, 'Closed orbits of fixed energy for singular Hamiltonian systems', Arch Rational Mech. Anal. 112 (1990), 339-362.

[3] A. Ambrosetti and V. Coti Zelati, Periodic solutions of singular lagrangian systems (Birkhäuser, Boston, 1993).

[4] U. Bessi and V. Coti Zelati, 'Symmetries and non-collision closed orbits for planar $N$-body type problems', Nonlinear Anal. 16 (1991), 587-598.

[5] V. Coti Zelati, 'A class of periodic solutions of the $N$-body problem', Celestial Mech. Dynam. Astronom. 46 (1989), 177-186.

[6] V. Coti Zelati, 'The periodic solutions of the $N$-body problems', Ann. Inst. H. Poincaré Anal. Non Linéaire 46 (1989), 177-186.

[7] E. Fadell and S. Husseini, 'Infinite cuplength in free loop spaces with an application to a problem of the $N$-body type', Ann. Inst. H. Poincaré Anal. Non Linéaire 9 (1992), 305-319.

[8] F. Giannoni and M. Degiovanni, 'Dynamical system with Newtonian type potentials', Ann. Scuola Norm. Sup. Pisa Cl. Sci. (4) 15 (1988), 305-319. 
[9] E.W. Van Groesen, 'Analytical min-max methods for Hamiltonian break orbits of prescribed energy', J. Math Anal. Appl. 132 (1988), 1-12.

[10] P. Majer and S. Terracini, 'Periodic solutions to some $N$-body type problems: The fixed energy case', Duke Math. J. 60 (1993), 683-697.

[11] E. Vitillaro, 'Noncollision periodic solutions of fixed energy for a symmetric $N$-body type problem', in Proceedings of the Workshop on Variational and Local Methods in the Study of Hamiltonian Systems (Trieste 1994) (World Scientific, River Edge, NJ, 1995), pp. 202-211.

[12] S.Q. Zhang, 'Multiple closed orbits of fixed energy for $N$-body-type problems with gravitational potentials', (preprint, 1993).

Department of Applied Mathematics

Chongqing University

Chongqing 630044

People's Republic of China 